PROFESIONALES Y HERRAMIENTAS PARA EL DESARROLLO LOCAL Y SUS SINERGIAS TERRITORIALES. EVALUACIÓN Y PROPUESTAS DE FUTURO IX Coloquio Nacional de Desarrollo Local del GTDL-AGE 

ANTONIO MARTÍNEZ PUCHE, XAVIER AMAT MONTESINOS, ISABEL SANCHO CARBONELL y DANIEL SANCHIZ CASTAÑO (EDS.)

\section{PROFESIONALES Y HERRAMIENTAS PARA EL DESARROLLO LOCAL Y SUS SINERGIAS TERRITORIALES. EVALUACIÓN Y PROPUESTAS DE FUTURO}

IX Coloquio Nacional de Desarrollo Local del GTDL-AGE

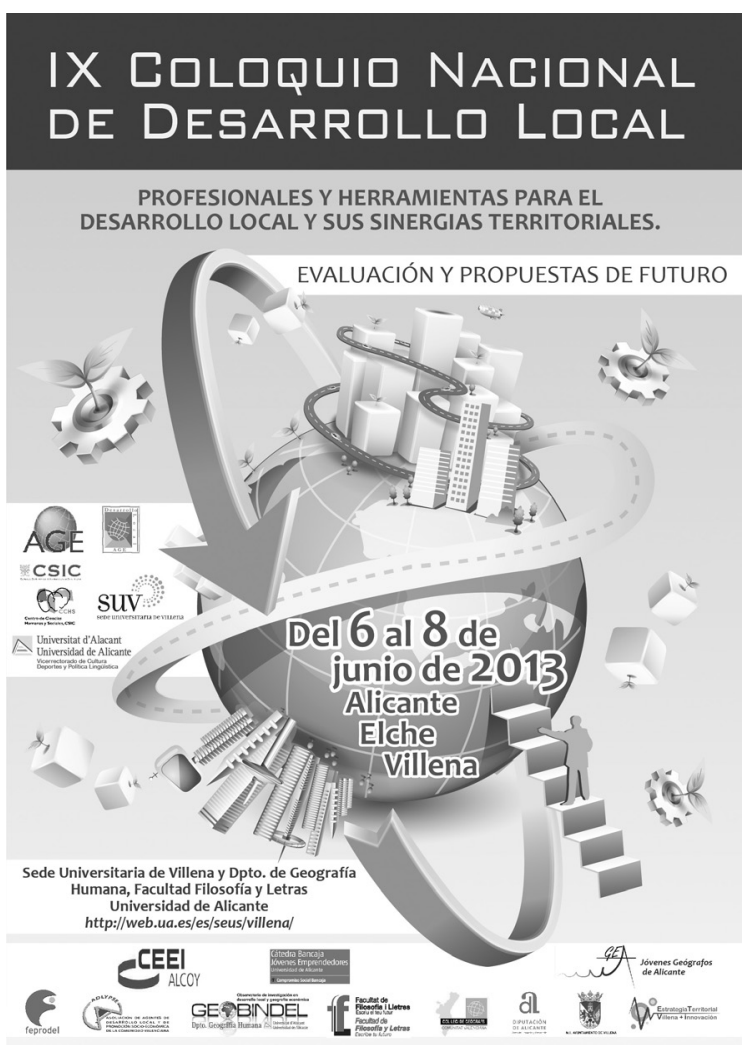


Este libro ha sido examinado y valorado por evaluadores ajenos a la Universidad de Alicante, con el fin de garantizar la calidad científica del mismo.

Publicacions de la Universitat d'Alacant

03690 Sant Vicent del Raspeig

Publicaciones@ua.es

http://publicaciones.ua.es

Telèfon: 965903480

(C) Antonio Martínez Puche, Xavier Amat Montesinos,

Isabel Sancho Carbonell y Daniel Sanchiz Castaño (eds.), 2016

(C) d'aquesta edició: Universitat d'Alacant

ISBN: 978-84-16724-00-0

Dipòsit legal: A 92-2016

Disseny de coberta: candela ink

Composició: Página Maestra (Miguel Ángel Sánchez Hernández)

Impressió i enquadernació: Guada Impresores

\section{unte \\ Unión de Editoriales
Universitarias Españolas \\ WWW.une.es
WWA}

Esta editorial es miembro de la UNE, cosa que garantiza la difusión y comercialización nacional y internacional de sus publicaciones.

Reservados todos los derechos. Cualquier forma de reproducción, distribución, comunicación pública o transformación de esta obra sólo puede ser realizada con la autorización de sus titulares, salvo excepción prevista por la ley. Diríjase a CEDRO (Centro Español de Derechos Repográficos, www.cedro.org) si necesita fotocopias o escanear algún fragmento de esta obra. 


\title{
DESARROLLO LOCAL: ¿UNA RESPUESTA A LOS DESAFÍOS DE LA RECUPERACIÓN?
}

\author{
Antonio Vázquez Barquero \\ Universidad Autónoma de Madrid \\ vazquez-barquero@uam.es
}

RESUMEN

Este artículo se pregunta si la política de desarrollo local es un buen instrumento para activar la recuperación económica en España, como lo fue en los años ochenta. Argumenta que las políticas de abajo hacia arriba siguen siendo adecuadas para mejorar la competitividad de los territorios. Pero, dado que las decisiones de inversión se toman en un entorno en el que convergen nuevos fenómenos como la globalización, las innovaciones tecnológicas y la desregulación, la política territorial será eficaz si se ejecuta con medidas adecuadas para resolver los problemas de cada territorio.

Palabras clave: Territorio, recuperación, economía española, espacios innovadores, desarrollo territorial.

\section{LOCAL DEVELOPMENT: A STRATEGY FOR FOSTERING ECONOMIC RECOVERY IN SPAIN?}

\section{Abstract}

This article focuses on discussing whether local development policy is a useful instrument for helping to overcome the economic crisis in Spain, as it did in the 1980s. It argues that the bottom-up approach continues to be adequate for improving the competitiveness of territories. But we must realize that the environment in which the investment decisions are made has changed. Globalization, technological innovation and economic deregulation require new policy tools to discover concrete solutions for the problems of specific territories and help foster their economic recovery.

Key words: Territory, recovery, Spanish economy, innovative spaces, territorial development. 


\section{INTRODUCCIÓN}

A principios de los años ochenta del siglo xx, se produjo un cambio de orientación en las políticas de desarrollo regional en Europa (VÁZQUEZ, 1999). Se reducen, paulatinamente, las políticas de "arriba hacia abajo", gestionadas por las administraciones centrales del estado y orientadas a la redistribución de la actividad económica y a la reducción de las disparidades regionales. Al mismo tiempo, comienza a tomar cuerpo el enfoque "de abajo hacia arriba" en el que las políticas están promovidas y gestionadas por los gobiernos locales y regionales, y están orientadas al desarrollo económico de ciudades y territorios concretos.

Este cambio tan significativo se puede entender como una respuesta de las comunidades locales a los desafíos que planteaba la reestructuración del sistema productivo europeo, inducida por el aumento de los precios de la energía, la desregulación en el ámbito público y el cambio social (BENNET, 1989; STÖRHR, 1990). En Europa las políticas de desarrollo local se difunden con rapidez en territorios cuya reestructuración productiva afecta a todo tipo de actividades (como la agricultura, el textil, el naval, la siderurgia, las manufacturas metálicas, los servicios), localizadas en ciudades y territorios con dimensiones y funciones muy diferentes (como las grandes metrópolis, las ciudades medias y pequeñas, las localidades de áreas rurales).

Dado que las características productivas, sociales e institucionales de los territorios eran diferentes, la política de desarrollo local tomó formas propias en cada uno de los países de la Unión Europea. En España, en concreto, a partir de principios de los años ochenta surgen y se difunden las experiencias de desarrollo local de la mano de los ayuntamientos democráticos. Las políticas de desarrollo local se consolidan en territorios cuyas empresas pierden competitividad en los mercados; y la reestructuración productiva afecta a actividades muy diversas (como las agrarias, pesqueras y agroalimentarias; el textil y los sectores industriales en declive; las actividades manufactureras en transformación), localizadas en ciudades y territorios con distintos niveles de desarrollo (VÁZQUEZ, 1993).

En el momento actual, el sistema productivo español atraviesa por un proceso de transformación de gran calado, asociado con la crisis económica que se ha hecho visible a finales de la década pasada. La crisis actual como las de otros ciclos económicos sigue un modelo especulativo (MINSKY, 1982; BORDO, 2008), tanto en su dimensión financiera como en la productiva. Después de un periodo en el que se produjeron burbujas en los mercados y el sobreendeudamiento de los operadores, se asiste al cierre de empresas en la construcción, en el sector manufacturero, y en los servicios financieros y comerciales. Pero el entorno en el que se produce la evolución del ciclo actual es diferente al que caracterizaba la dinámica de la economía en la década de los años ochenta del siglo pasado. 
Las empresas y los territorios donde se localizan, están en un escenario caracterizado por la convergencia de procesos como la globalización, la difusión de las tecnologías de la información, y la desregulación económica cuya interacción genera efectos no siempre bien conocidos. Por ello, la recuperación en el ciclo actual adquiere características propias, ya que las empresas toman sus decisiones de inversión y localización en un entorno específico de relaciones económicas, sociales y políticas, que se está ajustando a los nuevos procesos. De ahí que, la recuperación necesariamente tenga que apoyarse en las iniciativas específicas de cada territorio que fortalezcan las ventajas competitivas de las empresas locales.

La presente exposición argumenta que la política territorial sigue teniendo validez en la actualidad, pero sus objetivos e instrumentos es necesario adaptarlos para dar la respuesta adecuada a los desafíos que plantea la recuperación en cada territorio. Se analiza, en primer lugar, como durante la recesión ha comenzado a definirse el cambio estructural del sistema productivo, base de la recuperación. A continuación, después de señalar los rasgos principales del entorno en el que tiene lugar la recuperación, se discute cuáles son las características de las empresas y de los espacios innovadores, que están dando respuestas adecuadas a los desafíos que plantea el aumento de la competencia en los mercados. Las enseñanzas de la realidad ayudan a definir los objetivos y contenidos de las políticas territoriales para estimular a las empresas invertir y acelerar la recuperación económica. Se concluye señalando que el desarrollo territorial se fortalece cuando los actores públicos y privados combinan sus iniciativas y esfuerzos para transformar la economía y la sociedad.

\section{RECESIÓN Y CAMBIO ESTRUCTURAL}

Después del periodo de desarrollo industrial, de 1961 a 1975, la evolución del sistema productivo español se ha caracterizado por un complejo proceso de crecimiento y cambio estructural. La economía española ha crecido, en general, por encima de la media europea; entre 1992, inicio de la crisis de los años noventa, y 2007, inicio de la crisis actual, el PIB creció a una tasa anual superior al 3\%. Pero ha tenido una trayectoria inestable en la que se han producido fuertes ajustes de su sistema productivo, a medida que se integraba en la economía mundial. Los años ochenta del siglo pasado se caracterizaron por la desindustrialización y tercerización del sistema productivo, mientras que los noventa lo fueron por la internacionalización de la economía y la deslocalización de las actividades industriales a terceros países. El siglo XXI se inicia con la expansión de las actividades intensivas en trabajo, pero después de la crisis la recuperación plantea fuertes desafíos a las empresas innovadoras para liderar la nueva etapa de cambio estructural de la economía española.

Después de un periodo de crecimiento sostenido a principios de la década pasada (3,4\% anual del PIB durante 2002-2007), la economía española, como 
las de otros países avanzados, se ha enfrentado a una fuerte recesión, que se ha realizado en dos tiempos. Según el IMF (2013), el PIB se redujo en un 3,7 $\%$ en 2009 y en un $0,3 \%$ en 2010 (en la Eurozona la tasa de crecimiento del PIB fue del -4,4\% y del 2,0\%, respectivamente, y en los Estados Unidos pasó del $-3,1 \%$ al 2,4\%). Después de haber crecido un $0,4 \%$ en 2011 , se produjo, de nuevo, una reducción del PIB del 1,4\% en 2012, con un comportamiento análogo en la Eurozona (del 1,4\%, y del $-0,6 \%$, respectivamente), mientras que EEUU tuvo un crecimiento del PIB del 2,2\% en 2012, que sigue a un crecimiento del 1,8\% en 2011.

El ajuste productivo supuso una destrucción neta de empleo entre 2008 y 2013 de tres millones setecientos setenta mil ocupados, que llevó la tasa de paro al 25,1\% de la población activa en 2012 (INE, 2013). Esta caída del empleo conviene considerarla en términos de la evolución a largo plazo del mercado de trabajo de la economía española. En el periodo de auge anterior a la crisis se produjo un fuerte flujo migratorio procedente, sobre todo, de los países del este de Europa, norte de África y América Latina, de tal magnitud que la población extranjera registrada se multiplicó por diez entre 1996 (542.314 personas, 1,4\% de la población total) y 2010 (5.747.734 personas, $12,2 \%$ de la población total).

¿A qué actividades productivas afecta la recesión actual de la economía española? La crisis económica produjo una contracción, sobre todo, en la construcción y en la industria manufacturera. De los tres millones setecientos setenta mil empleos destruidos entre el primer trimestre de 2008 y el de 2013, el $43 \%$ corresponde a empleos de la construcción; el 26,4\% a las actividades manufactureras, en particular, a aquellas relacionadas con la producción de materiales de construcción (como la cerámica y el mármol) y a las actividades tradicionales (como el textil, calzado y mueble); pero también afectó a actividades como la electrónica de consumo, los equipamientos de oficina y telecomunicaciones, y la industria del automóvil ¿Cómo les fue a los servicios? El empleo se redujo en más de un millón en las actividades de servicios durante el periodo de recesión, entre el primer trimestre de 2008 y el de 2013, y afectó, sobre todo, a servicios públicos como la educación y la sanidad, pero también, a los bancarios y comerciales, en un proceso que no ha concluido.

Al analizar el efecto espacial del ajuste del sistema económico español destaca su asimetría, ya que afecta de manera desigual a las regiones y ciudades españolas. Una primera aproximación parecería indicar que sigue funcionando el viejo modelo de un norte y un centro con un sistema productivo más adaptado a los cambios tecnológicos y comerciales, y un sur que sigue un modelo más intensivo en trabajo. La tasa de paro (INE, 2013) superó la media nacional en las regiones del eje mediterráneo (con la excepción de Cataluña) en el primer trimestre de 2013, en Castilla la Mancha (31,5\%), Andalucía (36,9\%), Extremadura (35,6\%), y en las islas Baleares $(28,6 \%)$ y las Canarias $(34,3 \%)$; particularmente en territorios en los que el turismo es la actividad dominante y 
la construcción ha sido motor del crecimiento en las últimas décadas. La crisis ha tenido un impacto menor en las regiones del norte de la península (como Galicia, con un 22,4\%), del eje del valle del Ebro (Navarra, con el 19,0\%; Aragón con un 22,4\%; y la Rioja con el 19,0\%) y en aquellos territorios especializados en actividades intensivas en conocimiento y en servicios (como el País Vasco, con el 16,3\%, Cataluña con un 24,5\% y Madrid, con el 20,4\%).

¿Cuáles son los factores que explican el deterioro de la economía española a partir de finales de la década pasada? ¿Cómo se explica la pérdida de competitividad de las empresas españolas en los mercados nacionales e internacionales? ¿Se está produciendo un cambio estructural en sistema productivo español? ¿Por qué durante la crisis las regiones y territorios españoles tuvieron un comportamiento diferente? ¿Se debió a las características del entorno en el que las empresas realizaban su actividad?

En la base de la evolución del sistema productivo está el "efecto EURO" sobre la demanda. La entrada de España en la eurozona en 1999 fortaleció la integración económica de España en Europa, lo que aumentó sus mercados y produjo alteraciones importantes en su sistema financiero (TAMAMES, 2009; RECARTE, 2010). El sistema bancario (bancos y cajas), las organizaciones financieras, las empresas y las familias pudieron acceder a abundante crédito a bajos tipos de interés lo que activó la formación de una burbuja financiera; y el aumento del gasto público y privado generó el optimismo de los banqueros, políticos y ciudadanos. Las buenas condiciones crediticias proporcionaron liquidez creciente al sistema económico, lo que alimentó la burbuja inmobiliaria durante la fase de expansión del ciclo económico.

La demanda aumentó como consecuencia del crecimiento del empleo y de los salarios, y del aumento de las inversiones de las empresas, lo que afectó a las actividades productivas durante la fase de expansión del ciclo económico. Pero cuando se inició la recesión después del estallido de las burbujas financiera e inmobiliaria, el comportamiento de la demanda cambió y creó las condiciones para el deterioro de la actividad productiva (ALPHANDERY, 2012; ARIAS y COSTAS, 2011). Entre los mecanismos que lo facilitaron cabe destacar los siguientes:

- Ante todo, la sobrevaloración del mercado de la vivienda generó el sobre dimensionamiento de la construcción, que no pudo sostenerse cuando estalló la burbuja financiera, y las familias, promotores, constructores y agencias inmobiliarias no pudieron hacer frente a su endeudamiento. Ello produjo el cierre de empresas de la construcción, y de las actividades conexas como la cerámica y el mármol, lo que provocó el aumento del paro.

- Además, la reducción de las actividades industriales tradicionales se aceleró como consecuencia del aumento y de los cambios de la demanda interna durante la fase de expansión del ciclo. La desindustrialización 
se había iniciado a principios de los años noventa cuando la internacionalización de las actividades económicas había inducido la deslocalización de las actividades industriales como el textil, la confección, la alimentación, la química, la óptica y la automoción. El aumento de la renta durante el periodo de auge de la economía impulsó la demanda de productos de calidad y el crecimiento de las importaciones de productos transformados, lo que tuvo un efecto negativo en la demanda y en la producción manufacturera tradicional.

- El comportamiento de la demanda externa en la fase recesiva del ciclo, a su vez, ha impedido un deterioro más fuerte de la economía, debido, sin duda, a la caída de las importaciones inducida por la contracción de la demanda interna, pero, sobre todo, debido a la mejora de la competitividad. Entre 1998 y 2008, la economía española creció sin un aumento significativo de la productividad, ya que los costes laborales por unidad producida en la industria superaron en un $20 \%$ la media de la Eurozona, y fueron muy superiores a los de países como Alemania. Sin embargo, en los últimos cuatro años, la demanda externa ha mejorado progresivamente como consecuencia de la mejora de la competitividad, lo que ha neutralizado en parte el efecto de las fuerzas que afectaban negativamente al comportamiento del PIB, y ha contribuido a la reducción del déficit de la balanza comercial.

- Por último, el estallido de la burbuja financiera, a partir de 2007, en los EEUU afectó al sistema bancario europeo. El impacto sobre los bancos y las cajas de ahorro de España fue importante no solo por la interacción entre las dimensiones financiera y productiva en la evolución de la economía, sino sobre todo debido a la reacción tardía de las autoridades monetarias españolas y a las debilidades de las instituciones españolas y europeas. Fue preciso su rescate a través de nacionalizaciones, del estímulo a las uniones y fusiones de las entidades financieras, de la financiación de bancos y entidades con falta de liquidez y del apoyo a la recapitalización de los bancos con fondos públicos y privados. Su efecto difiere de unas comunidades a otras y, así, mientras los sistemas financieros del País Vasco y de Madrid se mantienen, el de Galicia se debilita.

Pero, cuando se profundiza en el análisis se observa que el cambio de la demanda de productos españoles ha propiciado la diversificación productiva de bienes y servicios en todo tipo de territorios: nuevos productos agrarios (como los vinos, la fruta, el aceite y los vegetales, producidos en Galicia, la Rioja, Andalucía, Valencia o Castilla), productos industriales reestructurados (la confección, las máquinas herramientas o el automóvil, en Galicia, el País Vasco, Navarra, Aragón y Cataluña), productos de alta tecnología (el aeroespacial, la microelectrónica, o la biotecnología, en Sevilla, Madrid y Barcelona), servi- 
cios avanzados a las empresas (marketing y diseño, en Madrid, Barcelona, A Coruña), nuevos servicios financieros, servicios culturales (museos en ciudades grandes, medianas y pequeñas) y actividades creativas (música, teatro). Se trata de la producción de bienes y servicios para los nuevos mercados nacionales e internacionales, lo que permite a los territorios en donde se localiza las empresas más dinámicas, adquirir ventajas competitivas como consecuencia de la adopción de innovaciones de producto, de proceso y de mercado.

\section{LA RECUPERACIÓN EN LOS ESPACIOS INNOVADORES}

El crecimiento económico y la creación de empleo se han convertido en la cuestión central de la recuperación económica en una situación en la que la demanda interna se ha reducido de manera sustancial y en la que el acceso de las empresas a la financiación bancaria es muy limitado. Sin la creación de empleo no es posible generar un aumento sostenido de la demanda y propiciar el ahorro necesario para reducir el endeudamiento privado y público y aumentar la inversión. La cuestión, sin embargo, no se plantea de la misma forma en todos los territorios, ya que tan solo en aquellos en los que el modelo de desarrollo está orientado a la mejora de la competitividad de las empresas en los mercados, se dan las condiciones para responder a los desafíos que plantea la recuperación económica.

La integración creciente de los mercados ha dinamizado las relaciones entre las empresas y territorios a través de los flujos e intercambios comerciales y financieros. En los territorios innovadores, durante décadas se ha fomentado el cambio estructural y el progreso económico y social, al incorporar innovaciones en la producción de bienes y servicios, al introducir cambios en la organización del sistema productivo, adecuar los espacios de producción a los flujos e intercambios en un mundo cada vez más integrado, y ajustar las reglas de juego y las instituciones a la nueva realidad de la economía y de la sociedad. Ante los desafíos que presenta el aumento de la competencia, las empresas más dinámicas han respondido a través de estrategias que han fortalecido su posicionamiento en los mercados; de tal forma que, cuando se produjo la crisis económica, los espacios donde se localizan las empresas innovadoras reunían un conjunto de factores que facilitan la recuperación.

El ciclo económico actual evoluciona en unas condiciones diferentes a las de los precedentes, ya que está marcado por cambios importantes en el funcionamiento del sistema económico internacional. La fase actual se caracteriza por la integración de los mercados internacionales y de los sistemas productivos, procesos que se suelen identificar con la globalización. El comercio mundial se multiplicó por cinco entre 1970 y 2000, y el comercio entre los países de Sur se multiplicó por diez durante las dos últimas décadas. La internacionalización de la producción se ha intensificado de tal manera que el stock de inversiones internacionales directas se multiplicó por dieciséis entre 1982 y 2007, las ven- 
tas de las EMN se multiplicaron por diez, y las inversiones extranjeras directas de las economías emergentes (como China, India, Brasil, Chile y Malasia) crecieron también de forma significativa.

Este impresionante cambio en los intercambios económicos internacionales se ha apoyado en la difusión de las tecnologías de la información (primer personal computer de IBM en 1981, surgimiento de la banda ancha para el transporte de la información en 1989, Internet en 1991), la desregulación económica de los países (liberalización de los mercados internacionales, privatización de las empresas públicas), las alianzas y cooperación de empresas a nivel internacional, y la creación de regiones económicas (Asean, Mercosur, Unión Europea). Sus efectos han dado lugar a la reestructuración de las actividades productivas y la aparición de actividades más avanzadas en la industria (biotecnología, electrónica, farmacéutica) y en los servicios (financieros, de gestión, culturales, de ocio). La transformación del sistema productivo, liderada por empresas de dimensiones muy diferentes, localizadas en territorios y ciudades con diferentes niveles de desarrollo, está generando, a su vez, una nueva geografía del desarrollo económico a escala internacional y dentro de las economías nacionales (OECD, 2010).

Así pues, el proceso de transformación económica está dando lugar a una nueva organización espacial de la producción, favorecida por el aumento de los flujos económicos, financieros y culturales, y estimulada por las nuevas tecnologías, que se aprecia con claridad en los países de industrialización tardía como España. La nueva organización espacial del desarrollo es el resultado de un proceso impulsado por las estrategias de las empresas y territorios que durante décadas han liderado la evolución y cambio del sistema productivo (VÁZQUEZ, 2011). Se trata de un proceso de transformación que no es uniforme en todos los territorios, ya que se produce en lugares y espacios urbanos concretos, que son estratégicos en las decisiones de inversión y de localización de los agentes económicos, públicos y privados. Son espacios motores de los procesos de desarrollo, cuyas empresas y organizaciones han respondido más eficientemente a los desafíos que plantea el aumento de la competencia en los mercados, dado que ellas son capaces de instrumentar sus decisiones estratégicas a través de sistemas creativos de producción, de organización y de mercado.

En los territorios innovadores de España se puede observar cómo se han ido creando las condiciones para la recuperación económica, dando lugar a diversos modelos de desarrollo territorial. En Arteixo, municipio del área metropolitana de A Coruña (con 386 mil habitantes y una tasa de paro provincial del 15,6\% para un total de 1.139 mil habitantes), surgió en 1975 Zara, una empresa capaz de organizar una red global de empresas productoras y comercializadoras de prendas de vestir y moda, "fast fashion" (ERIKSSON y JONSSON, 2011). En Getafe, municipio (con 171 mil habitantes) del área metropolitana de Madrid (con una tasa de paro de la comunidad autónoma del 15,4\% para una 
población de seis millones cuatro cientos mil habitantes), una ciudad global en formación (TAYLOR, 2004), se ha creado una red de empresas especializadas en la producción de partes de aviones comercializados por EADS-CASA y Airbus-España. En Mondragón (con 22.064 habitantes), una ciudad articulada al sistema de ciudades de Guipúzcoa (con 705 mil habitantes y un $9 \%$ de paro), la Unión Cooperativa se internacionaliza a principios de los años noventa del siglo pasado (ELIO, 2004).

En los espacios innovadores se ha producido un fuerte proceso de cambio estructural en las últimas décadas, caracterizado por la introducción de conocimiento en las actividades productivas de la mano de empresas innovadoras y creativas. La creación de Zara en 1975 y del grupo de Inditex en 1985 contribuyó a la transformación de A Coruña en una ciudad creativa. La localización en Arteixo de la planta de Zara, de los servicios de dirección y el centro de diseño del grupo Inditex y del centro logístico (Plataforma Europa), ha reforzado el sistema local de innovación vinculado a la universidad y, por otro lado, el desarrollo de servicios culturales y de ocio (PRECEDO, 2007). La dinámica reciente de Madrid se generó alrededor de actividades industriales de alta tecnología, como el clúster aeroespacial, de la internacionalización de sus servicios financieros, de la concentración de los servicios a las empresas y de los servicios avanzados de conocimiento (telemática, ingeniería del software, laboratorios); y se apoya en un potente sistema regional de innovación, formado por las universidades públicas y privadas, los parques científicos y tecnológicos y por organizaciones especializadas en la producción, transferencia y gestión del conocimiento (CAICEDO, 2010). La Cooperativa de Mondragón, creada en 1956, ha dado paso a la Corporación Mondragón, con cooperativas especializadas en actividades industriales, y en servicios financieros y de distribución, en cuyo centro está la creación de un sistema local de innovación, articulado a través de las oficinas de I+D de las cooperativas, los planes de ciencia corporativos, y la vinculación con la Universidad.

En los espacios innovadores la organización de la producción se hace, además, cada vez más flexible, debido tanto a los cambios en la organización de las grandes empresas como a la formación de redes de empresas y a la proliferación de los acuerdos entre empresas. Inditex ofrece un buen ejemplo de una cadena de valor internacional que permite una respuesta rápida de la producción a los cambios de la demanda. Tiene un modelo de organización vertical que estimula la integración de buena parte de las operaciones de materias primas, el proceso productivo (diseño, corte, fabricación intensiva en capital, control de calidad, planchado y embalaje) y la distribución. Su sistema de producción flexible permite dar una respuesta rápida a las demandas de los clientes, ya que el aprovisionamiento y producción se realiza cerca de los puestos de venta, y, además, el modelo de organización estimula la diferenciación de la producción, también, mediante la autonomía de las marcas comerciales (como 
Zara, Massimo Dutti, Pull \& Bear, Berska, Stradivarius y Oysho). A su vez, el clúster de la industria aeronáutica de Getafe forma parte de una red de clusters, cuyas empresas operan en Asia, América y Europa, formando una cadena de valor internacional que permite la circulación de innovación y conocimiento en toda la red de empresas (ALFONSO y VÁZQUEZ, 2010). Por último, la internacionalización de la producción de la Corporación de Mondragón ha requerido un cambio importante en la organización a partir de 1991, pasando de un modelo de agrupación territorial, a un sistema de estructuración sectorial, según los tipos de bienes producidos en cada cooperativa; pero, la Corporación ha mantenido una organización federativa en la que cada cooperativa mantiene su soberanía en la toma de decisiones.

Finalmente, nuevas formas de organización del territorio han impulsado la formación y desarrollo de plataformas creativas que facilitan la competitividad de las empresas en los mercados nacionales e internacionales. Arteixo, Getafe y Mondragón forman parte de regiones urbanas policéntricas, formadas por una red de ciudades con una fuerte especialización productiva y una gran integración laboral y social, que se apoya en una potente infraestructura de transporte y comunicaciones (VÁZQUEZ et al. 2009). Además, en el caso de Inditex, las TICs juegan un papel estratégico en los flujos de información y conocimiento entre las empresas y los lugares, ya que a través de las tecnologías de la información más de seis mil tiendas se relacionan con los centros logísticos a quienes informan de los cambios en la demanda y transmiten sus necesidades de producto de manera sistemática; lo que permite a Zara abastecer sus tiendas con regularidad dos veces por semana a través del transporte por carretera en Europa, y aéreo en el resto del mundo.

\section{Sobre las POlíticas terRITORIALES DE LA RECUPERACióN}

La actual recesión de la economía española tiene un carácter singular ya que el ciclo económico evoluciona dentro de un entorno económico, político e institucional diferente al de los anteriores, como se acaba de ver. La recuperación requiere, por lo tanto, la adaptación de las estrategias empresariales a los cambios del entorno competitivo. Desde la perspectiva del pensamiento schumpeteriano, la capacidad innovadora y la difusión de las innovaciones y el conocimiento son mecanismos que afectan al proceso de acumulación de capital y condicionan la evolución del ciclo. Por lo tanto, la recuperación económica se apoya necesariamente en la creatividad y la capacidad emprendedora de las empresas que toman las decisiones de inversión, si bien su ritmo de crecimiento está condicionado, entre otros factores, por la adaptación de las instituciones a las nuevas condiciones del entorno económico y social, y por las políticas de recuperación específicas de cada territorio. 


\subsection{Recuperación y territorio}

Si, como señala SCHUMPETER (1934; 2005), los factores determinantes del desarrollo son las empresas, las innovaciones y las instituciones, puede entenderse que se trata de un proceso cuyas dimensiones microeconómica y territorial son determinantes. El ciclo económico, a su vez, haría referencia a los cambios de los mecanismos endógenos del sistema económico, que provocan su transformación en otros más adecuados para abordar la nueva dinámica de la economía y la sociedad. Por ello, el desarrollo puede entenderse como un fenómeno territorial en el que los actores que toman las decisiones de inversión están inmersos en el sistema de relaciones institucionales, culturales y sociales que caracterizan a cada territorio. Y, como se ha visto en el apartado anterior, las transformaciones económicas y productivas tienen lugar en los territorios en donde se localizan las empresas innovadoras y las actividades motoras del desarrollo (VÁZQUEZ, 2011).

La especialización productiva de las regiones y territorios se ha ido construyendo históricamente, en función del potencial de crecimiento natural existente en el territorio y del sistema de relaciones económicas y políticas que se han desarrollado con otros territorios. Por lo tanto, la especialización productiva de las regiones y territorios depende de las ventajas comparativas que han ido adquiriendo a medida que se incorporaba nuevo conocimiento en el sistema productivo y se transformaban las instituciones. Pero no basta con las ventajas naturales, basadas en los recursos existentes en el territorio en un momento histórico determinado, sino que, como señala PORTER (1990 y 1998), es necesario generar ventajas competitivas invirtiendo en factores avanzados que incorporan conocimiento en los recursos naturales y humanos, en las infraestructuras y los sistemas urbanos, y en la organización productiva y el sistema empresarial.

Se puede construir la escalera de las ventajas comparativas de los territorios (MEIER, 2005), en función de la composición del comercio entre los territorios e identificar cual es la posición de cada territorio en cada momento histórico. En la parte baja de la escalera, se trataría de la ventaja comparativa natural, relacionada con la producción agraria y de bienes intensivos en mano de obra como el textil, que analizó David Ricardo; mientras que en la parte superior estarían aquellos territorios que tienen ventajas comparativas adquiridas como consecuencia de la introducción de innovaciones y conocimiento en los productos y procesos productivos, según indican KRUGMAN (1980) y PORTER (1990). Como se ha argumentado anteriormente, la recuperación económica pasa por que las empresas introduzcan innovaciones que transformen el sistema productivo de manera que los territorios mejoren sus ventajas competitivas en los mercados de productos y factores.

Pero, la recuperación económica puede tener efectos no previstos sobre el empleo y el medioambiente, e incidir negativamente en la cohesión social y en 
la conservación de los recursos naturales y en el medioambiente. Ante todo, la recuperación puede tener un efecto negativo sobre el empleo en territorios cuyas empresas introducen innovaciones que ahorran trabajo como consecuencia de que el aumento de los costes salariales ha reducido su competitividad durante las últimas décadas (SINAI, 2010). La alternativa al empleo sería la utilización de nuevas tecnologías, como la robótica y las TICs, que pueden implicar en algunas actividades industriales la reducción del empleo, en ocasiones por encima del $80 \%$. Si a ello se añade que las empresas pueden haber organizado su actividad productiva a través de cadenas globales de valor y deslocalizan parte de su actividad productiva, la incertidumbre sobre la recuperación y sus efectos sobre la creación de empleo aumenta.

Finalmente, los propios mecanismos de la economía de mercado estimulan a las empresas a buscar el éxito económico y los beneficios de sus inversiones a corto plazo, sin considerar sus efectos sobre el progreso social y el medioambiente, en la idea de que ello concierne a las políticas públicas y no a las inversiones de las empresas. PORTER y KRAMER (2011) argumentan, sin embargo, que las empresas fortalecerían su competitividad si sus estrategias y acciones contribuyeran a mejorar las condiciones económicas y sociales de los territorios y de las comunidades donde localizan su actividad productiva y comercializan sus productos, ya que el entorno económico, social e institucional de los territorios condicionan el éxito de sus inversiones.

Por ello, proponen una nueva conceptualización del valor generado por las empresas, en el sentido de que además de tener en cuenta sus beneficios consideren los costes de todo tipo que genera en su actividad. La creación de valor compartido permite identificar las conexiones entre progreso económico y social ya que las empresas toman sus decisiones dentro del contexto económico, social e institucional de los lugares donde realizan su actividad. Es decir, la adopción y difusión de las innovaciones y, por lo tanto, la mejora de la productividad es un fenómeno localizado en territorios concretos. Por ello, Porter y Kramer proponen que las empresas creen valor económico creando valor social ya que al conectar el éxito de las empresas con el avance de la sociedad se gana en eficiencia, y se amplían los mercados.

\subsection{Demanda y actividad productiva de los territorios}

Así pues, la recuperación puede entenderse como un fenómeno territorial en el que las decisiones de inversión y de localización están condicionadas por la dinámica del entorno de la actividad empresarial. La integración creciente de los territorios en el sistema económico internacional aumenta la competencia en los mercados y altera las características de la demanda de sus productos, lo que condiciona la estrategia de las empresas y territorios, los objetivos, la organización de la producción, la adopción de innovaciones, los productos y los 
mercados. De ahí que las políticas territoriales para la recuperación adquieran nuevas dimensiones con respecto a las políticas de desarrollo local de los años ochenta.

El proceso de globalización está impulsando la formación de cadenas de valor y clusters, favoreciendo la formación de redes de ciudades y estimulando la conexión entre empresas y ciudades a través de la potenciación de las infraestructuras de transporte y comunicaciones, que incorporan las nuevas tecnologías de la información y las comunicaciones. Surgen y se consolidan las regiones policéntricas, como consecuencia de que la diversidad productiva aparece en lugares y espacios urbanos cercanos conectados entre sí, entre los que se intercambian factores productivos, bienes y servicios. ¿Cuál ha sido el impacto de estas transformaciones en el modelo de organización territorial de España? El panorama actual de la organización del territorio en España se caracteriza por un fuerte desarrollo urbano en el que se aprecia el surgimiento y la consolidación progresiva de regiones policéntricas, como puedan ser el País Vasco, Galicia o Madrid, cada una de las cuales tiene especificaciones propias (MINISTERIO DE LA VIVIENDA, 2004; PRECEDO y MÍGUEZ, 2007; VÁZQUEZ et al. 2009).

Estas empresas y territorios producen bienes y servicios para los mercados nacionales e internacionales que se han diversificado de manera singular en los últimos años. Este es un fenómeno que se estima que continuara en las próximas décadas debido al hecho de que la integración económica y el progreso tecnológico están propiciando un aumento progresivo de la riqueza en los países emergentes y en los países de desarrollo tardío, alterando de manera sustancial su posición a nivel mundial (OECD, 2010). El crecimiento de la renta ha sido muy diferente de unos países a otros de manera que los países que convergen han pasado de ser 12 en los años noventa del siglo pasado a 65 en la primera década de este siglo y que los países pobres se han reducido pasando de cincuenta y cinco en los noventa a veinticinco en la última década; si bien la desigualdad se mantiene en niveles elevados, alrededor de los 70 puntos del Gini (MILANOVIC, 2011).

El aumento de los niveles de renta de los países ha dado un fuerte impulso a la demanda interna y a la demanda internacional que se prevé continuará en los próximos años, si se considera como indicador el aumento de la clase media en el mundo (KHARAS, 2010). Según las estimaciones realizadas el Centro de Desarrollo de la OCDE, la clase media mundial alcanzó mil ochocientos millones de habitantes en 2009. En las próximas décadas el tamaño de la clase media mundial seguirá aumentando y llegará a tres mil doscientos millones en 2020 y a cuatro mil novecientos millones en 2030. Como consecuencia el gasto de la clase media mundial se estima que pasará de 21.278 millones de dólares (a precios de 2005) en 2009 a 55.680 en 2030; pero el aumento tendrá lugar sobre todo en los países asiáticos cuyo gasto representaba el $23 \%$ del gasto mundial 
en 2009 y se estima que significará el 59\% en 2030, mientras disminuye en Europa (del 38\% al 20\%) y en Norte América (del 26\% al 10\%).

No se conoce el efecto que tendrá el aumento de la demanda y el cambio de su distribución en el sistema productivo, ya que el gran crecimiento de la clase media de Asia, y específicamente de China e India, y la reducción relativa de la de los Estados Unidos tendrá una incidencia desconocida al ser diferentes la propensión al consumo y la preferencia de productos de los países asiáticos, y de los países europeos y los norteamericanos. Sin duda que entre los productos finales estarán los automóviles, televisores, frigoríficos y teléfonos móviles, que, en la actualidad, tienen incluso una difusión creciente en los países africanos. En todo caso, las características de la producción se están transformando ya que, como se indicó anteriormente, las ventajas comparativas adquiridas son cada vez más frecuentes en todo tipo de países cuando la demanda interna y la demanda internacional se expanden.

En este sentido cabe señalar, además, que los servicios han ido adquiriendo una fuerte presencia en la producción manufacturera. Como señala BARO (2013), en las últimas décadas se han producido grandes transformaciones en las actividades industriales ya que se ha generado una sinergia creciente entre manufactura y servicios, de manera que los servicios entran a formar una parte, cada vez mas importante en la producción manufacturera, a través del diseño, de la I+D, de la marca, y de los servicios técnicos e ingenieriles. En las actividades agrarias e industriales, los servicios a la producción están cada vez mas externalizados, y la actividad manufacturera se lleva a cabo a través de servicios incorporados a la producción en función de las relaciones existentes en las cadenas de valor, que pueden ser de signo muy diverso (GEREFFI, 1999).

La cuestión que se discute supone una revisión del concepto de actividad industrial. Cuando se habla de industria se hace referencia a la transformación de materias primas y de productos semielaborados en bienes materiales a los que se incorporan los servicios a la producción como son los de transporte y los servicios comerciales (FLACHER y PALLETAN, 2007). Pero, en la actualidad, se va mas allá ya que se trata, también, de la incorporación a la producción de bienes de los servicios de asistencia técnica, de adaptación de las innovaciones, y de los servicios financieros, a través de las nuevas formas de organización de la producción y de intercambio entre empresas, que ha estimulado la globalización del sistema productivo (SCHMITZ, 2007).

\subsection{Instrumentos para la recuperación en los territorios}

Por ello, la recuperación de la economía española precisa de un conjunto de medidas que activen la economía real y estimulen el aumento de la productividad y de la competitividad. En un entorno nuevo como el actual, las iniciativas de política económica deberían de tener en cuenta el cambio de la geografía de la producción y del desarrollo, adoptando una estrategia que enfocara el ajuste 
productivo con una visión territorial que potenciara soluciones concretas para abordar los problemas de territorios específicos, apoyándose en los factores innovadores, en sus potencialidades y sus ventajas competitivas. La definición y gestión de las acciones necesitaría articular las políticas públicas con las iniciativas de los agentes económicos dentro de un nuevo marco institucional, en el que las decisiones de inversión de los actores públicos y privados se ajustaran a una nueva regulación, y se reforzara la participación de la sociedad civil (VÁZQUEZ, 2010).

La visión desde el territorio de la reestructuración productiva sugiere desarrollar iniciativas que permitan mejorar la competitividad de las empresas, los clústeres y los sistemas productivos locales. Cada territorio tiene su propia trayectoria, lo que condiciona su dinámica productiva, si bien comparte con los demás, los objetivos de favorecer el surgimiento y desarrollo de las empresas, aumentar la flexibilidad del sistema productivo, facilitar el acceso a la financiación, potenciar y fomentar la difusión de las innovaciones en las empresas y en la sociedad. En esta línea de actuación el desarrollo industrial precisa de un fuerte apoyo público a través de políticas cuyo objetivo sea estimular la reconversión industrial, para lo que son necesarios instrumentos como las incubadoras de empresas y los centros de empresas e innovación, que impulsen la capacidad emprendedora de grupos sociales, como son los jóvenes, que se han convertido en público/objetivo de la política económica e industrial.

Pero, no siempre las iniciativas de desarrollo local que tuvieron éxito en las décadas pasadas son las más adecuadas para estimular la recuperación económica. En los años ochenta, después de la crisis de la gran empresa fordista, uno de los objetivo era la formación y desarrollo de clústeres y de distritos industriales, ya que se entendía que eran un estímulo para la especialización productiva de las pequeñas empresas en el tejido productivo, y la creación de economías de escala; las pequeñas empresas de los distritos industriales aseguraban una mayor flexibilidad al sistema productivo y contribuían a mejorar la productividad y la competitividad. Pero, el objetivo estratégico de la política territorial ha cambiado, ya que la recuperación productiva se apoya en la diferenciación de la producción y en la creación de nuevos productos, por lo que las pequeñas empresas tradicionales han perdido el papel estratégico que tuvieron en los años ochenta y noventa ya que su escala les limita la capacidad para innovar y para competir en los mercados globales (DRAGHI, 2012).

Las iniciativas orientadas a la creación y difusión de las innovaciones, por su parte, son determinantes en los procesos de reestructuración productiva y ponen a la disposición de las empresas, servicios que refuerzan sus capacidades competitivas. A su vez, la cooperación entre la empresa, los centros de investigación y desarrollo, y las universidades permiten estimular el surgimiento y desarrollo de clústeres y sistemas productivos especializados en actividades de alta tecnología, que pueden fructificar generando la atracción de proyectos intensivos en conoci- 
miento lo que estimula el cambio estructural hacia actividades cuyas producciones son valoradas en los nuevos mercados (ASHEIN, 2010).

La visión territorial del desarrollo señala, además, que el proceso de ajuste y la recuperación económica se ven favorecidos en aquellos territorios, cuya organización espacial y productiva obedece a un modelo policéntrico (HALL y PAIN, 2006). Por un lado, las regiones urbanas policéntricas tienen un tejido urbano más diversificado, lo que les permite neutralizar los efectos negativos de la crisis sobre partes de su tejido productivo, y responder de forma más articulada a los desafíos que plantea la restructuración productiva. En el caso de los territorios, sin embargo, en los que predomina la concentración espacial de las actividades, aunque puedan ser más eficientes y competitivas en los momentos de auge, son más frágiles en los periodos de recesión.

Por último, en los territorios bien articulados como las regiones urbanas policéntricas son más fáciles las respuestas coordinadas de los actores públicos y privados que toman las decisiones de inversión en el territorio. La cooperación entre los agentes económicos y sociales y entre las ciudades y localidades facilita la combinación y coordinación de las acciones, lo que las hace más eficaces, y contribuye a la realización de las transformaciones necesarias para reactivar la economía y aumentar la competitividad de las empresas y territorios, y estimular, así, el desarrollo sostenible.

En definitiva, el desarrollo endógeno de los territorios se ha convertido, sin duda, en un instrumento para favorecer la recuperación económica. En tiempos de incertidumbre como los actuales, la recuperación productiva requiere una reorientación de las relaciones entre las élites políticas y económicas. Ambas entienden que el aumento de la competitividad de las empresas es clave en los procesos de desarrollo económico y que los frutos de la actividad productiva deben de redundar en beneficio de la sociedad. Pero, las estrategias de las administraciones y de las empresas serían más eficaces si las medidas macroeconómicas y las de apoyo a la actividad productiva, como la política territorial, se articularan con las iniciativas empresariales, y la gestión del desarrollo de los territorios se apoyara en una efectiva alianza público-privada.

\section{Comentarios Finales}

En tiempos como los actuales, las políticas de abajo hacia arriba siguen siendo estratégicas para estimular la recuperación de la economía y el bienestar social. Pero, las estrategias, los objetivos, las acciones y la gestión tienen que adaptarse al entorno en el que las empresas y territorios toman sus decisiones de inversión, ya que se trata de un proceso en el que las interrelaciones entre las innovaciones tecnológicas, la integración económica internacional y la desregulación tienen efectos cambiantes y, en gran medida, inesperados. Por ello, la política territorial necesariamente ha de ir más allá de las políticas de desarrollo local tal como se instrumentalizaron en los años ochenta y noventa del siglo pasado. 
El actor decisivo sigue siendo el territorio, entendido como un agente de transformación y no como un mero soporte de los recursos y de las actividades económicas, ya que las decisiones de los ciudadanos, las empresas, las organizaciones sociales, los centros de formación y de investigación, y los organismos públicos, determinan el desarrollo de la economía y de la sociedad. Como se ha indicado anteriormente, el concepto de territorio que se utiliza no lo definen los límites administrativos, como los de las comunidades autónomas o los de los municipios, ya que considera espacios que obedecen a otros criterios como son las regiones urbanas policéntricas, y, además se tiene en cuenta que los sistemas productivos territoriales pueden estar integrados con los de otros territorios, por lo que en muchos casos es necesario considerar la dimensión internacional.

La apertura de los mercados productivos y financieros y la mejora de los transportes y las comunicaciones, han estimulado el aumento de la competencia y han ampliado la demanda de los productos locales. Por ello, los territorios y sus empresas pueden continuar atendiendo la demanda interna con sus productos, en concurrencia con los procedentes de otros territorios. Pero, la integración económica a través de acuerdos, como los de la Union Europea, y la reducción de las barreras comerciales han ampliado los mercados de los productos locales. Por ello, los territorios utilizan su potencial de desarrollo para atender las demandas de sus productos en mercados específicos.

De esta forma, la adopción de innovaciones se ha convertido en el desafío de las empresas y territorios, ya que permitirá completar la reconversión industrial que se inició durante el periodo de auge del ciclo económico. La competencia en los mercados y los cambios en la demanda interna y externa estimulan a las empresas a adoptar y adaptar el conocimiento científico y combinarlo con las mutaciones endógenas de las tecnologías utilizadas por las empresas; pero, además, la demanda de bienes industriales hay que satisfacerla con productos que combinan bienes materiales con servicios a la producción, debido a los cambios en los modelos de organización de las empresas.

Para que las estrategias y las acciones de desarrollo territorial puedan definirse y ejecutarse es necesario contar con la financiación pública y privada que permita a las empresas ejecutar sus decisiones de inversión y con las reglas de juego e instituciones que lo hagan viable. El rescate al que ha estado sometido el sistema bancario y financiero español no ha dado los resultados esperados por el momento y las empresas siguen encontrando dificultades para encontrar financiación, incluyendo las líneas de crédito del Instituto de Crédito Oficial para PYMEs que son solventes, innovadoras y se internacionalizan. Por otro lado, las administraciones públicas, como consecuencia del aumento continuo de la deuda pública desde el inicio de la recesión, que, en el momento actual, se acerca al 90\% del PIB, han dado prioridad al control del déficit público, lo que implica la reducción de los gastos públicos, incluyendo los de las Comunidades Autónomas y de los Municipios, lo que limita los recursos disponibles para la política territorial. 
Pero, para poder impulsar la recuperación económica con la participación de tal diversidad de actores es necesario introducir cambios en las organizaciones y las instituciones. La recuperación de la economía española se vería estimulada si la Unión Europea ampliara sus competencias, de manera que además de dirigir la política monetaria común, adoptara una regulación uniforme para el sistema financiero de la Unión y pusiera en marcha una política fiscal común. Pero, la recuperación de la economía española necesita, también, cambios institucionales internos, como la puesta en marcha de formas de gobierno más cercanas a los intereses territoriales, las reformas en el sistema financiero y en el mercado de trabajo, y el reforzamiento de las relaciones entre los actores públicos y privados que favorezcan la acción conjunta de quienes asumen y condicionan la toma de decisiones de inversión y de localización. Todo ello contribuiría, sin duda, a hacer más eficaces las medidas y las políticas y, en definitiva favorecería la restructuración productiva y la recuperación económica.

Para superar las restricciones financieras e institucionales actuales nada mejor que inspirarse en el comportamiento de los actores locales en el momento de surgimiento de la política de desarrollo local, cuando las empresas y territorios aportaron sus propios recursos, y crearon los mecanismos necesarios para definir y ejecutar, por primera vez, políticas económicas territoriales. En realidad, el desarrollo territorial y, por lo tanto, la recuperación económica precisa que las empresas impulsen el cambio de las relaciones entre la sociedad y las empresas y se pongan de acuerdo para transformar la economía y la sociedad.

\section{BIBLIOGRAFÍA}

ALFONSO, J. y VÁZQUEZ BARQUERO, A. (2010): "Networking and innovation: lessons from the aeronautical cluster of Madrid", Int. J. of Technology Management, $\mathrm{n}^{\circ}$ 50, p. 337-355.

ALPHANDERY, E. (2012): "The Euro Crisis", European Issues, nº. 240, p 1-13. Fundation Robert Schuman.

ARIAS, X. C. y COSTAS, A. (2011): La torre de la arrogancia. Políticas y mercados después de la tormenta, Barcelona, Editorial Planeta.

ASHEIM, B. (2010): "Nueva política de innovación: cómo combinar un enfoque científico con un enfoque orientado al usuario", en Parrilli, M.D. (coord.) Innovación y aprendizaje: Lecciones para el diseño de políticas, San Sebastián y Bilbao, Orkestra-Innobasque, p. 102-112.

BARO TOMAS, E. (2013): “¿Desindustrialización o metamorfosis de la industria? La nueva relación entre las actividades manufactureras y terciarias", Economia Industrial, n ${ }^{\circ}$ 387, p. 33-47.

BENNET, R. (1989): "Local economy and employment and development strategies: An analysis for LEDA Areas", LEDA Report, Brussels, European Commission. 
BORDO, D. M. (2008): "An historical perspective on the crisis of 2007-2009". Working paper, $\mathrm{n}^{\circ}$ 14569, Cambridge, Mass., National Bureau of Economic Research.

CAICEDO, H. (2010): "Análisis de la contribución del sistema regional de innovación en el desarrollo de las ciudades regionales globales: el caso de Madrid", Trabajo fin de máster, Máster en Economía y Gestión de la Innovación, Universidad Autónoma de Madrid.

DRAGHI, M (2012): "Crecita, benessere e compiti dell'economia politica", en Canullo, G. y Pettenati, P. (coord.), Sviluppo Economico e Benessere, Napoli, Edizioni Schientifiche Italiane, p. 41-51.

ELIO, E. (2004): "MCC, el paradigma del desarrollo del primer grupo industrial en España a través del cooperativismo de trabajo asociado", Mediterráneo Económico, no. 6, p. 335-347.

ERIKSSON, C. y JONSSON, S. (2011): "Inditex. A company analysis with focus on growth". Bachelor Thesis in Business Economics. University of Gothenburg.

FLACHER, D. y PALLETAN, J. (2007): «Le concept d'industrie et sa mesure: origines, limites et perspectives. Une application à l'étude des mutations industrielles », Economie et Statistique, nº. 405-406, p. 13-46.

GEREFFI, G. (1999): "International trade and industrial upgrading in the apparel commodity chain", Journal of International Economics, $\mathrm{n}^{\circ} .48$, p. 37-70.

HALL, P. y PAIN, K. (2006): The Polycentric Metropolis. Learning from Megacity Regions in Europe, London, Earthescan.

IMF (2013): World Economic Outlook. April 2013, Washington, International Monetary Fund.

INE (2013): Encuesta de Población Activa. Primer trimestre de 2013, Madrid, Instituto Nacional de Estadística.

KHARAS, H. (2010): "The emerging middle class in developing countries", Working paper, $\mathrm{n}^{\circ}$. 285, Paris, OECD Development Centre.

KRUGMAN, P. (1980): "Scale Economies Differentiation and the Pattern of Trade", American Economic Review, no. 70, p. 950-959.

MEIER, M. G. (2005): Biography of a Subject: An Evolution of Development Economics, New York, Oxford University Press.

MILANOVIC, B. (2011): "Income inequality has risen over the past quarter century instead of falling as expected", Finance and Development, September, p. 6-11.

MINISTERIO DE LA VIVIENDA (2004): Atlas Estadístico de las Áreas Urbanas en España, Dirección General de Suelo y Políticas Urbanas, http://atlas.vivienda.es/

MINSKY, H. (1982): Can "It" Happen Again? Essays on Instability and Finance, New York, Sharpe. 
Antonio Vázquez Barquero

OECD (2010): Perspectives on Global Development 2010. Shifting Wealth, Paris, OECD Development Center.

PORTER, M. (1990): The Competitive Advantage of Nations, New York, Free Press.

PORTER, M. (1998): "Clusters and the New Economics of Competition", Harvard Business Review, November-December, p. 77-90.

PORTER, M. y KRAMER, M. (2011): "Creating Shared Value", Harvard Business Review, January- February, p. 1-17.

PRECEDO LEDO, A. (2007): El Área Metropolitana de A Coruña: Una Metrópoli Euroatlántica, A Coruña, Editorial Deputación Provincial da Coruña.

PRECEDO LEDO, A. y MÍGUEZ IGLESIAS, A. (2007): "Policentrismo urbano y ajuste del sistema de ciudades en Galicia”, Xeográfica. Revista de Xeografía, Territorio e Medio Ambiente, no. 7, p. 75-93.

RECARTE, A. (2010): El desmoronamiento de España. La salida de la crisis y la política de reformas, Madrid, La esfera de los libros.

SCHMITZ, H. (2007): "Regional Systems and global chains", en SCOTT, J. A. y GAROFOLI, G. (coord.) Development on the Ground, Abington, Routledge, p. 322-339.

SCHUMPETER, J. A. (1934): The Theory of Economic Development, Cambridge, Mass., Harvard University Press, (1 $1^{a}$ edición en alemán, 1911).

SCHUMPETER, J. A. (2005): "Development", The Journal of Economic Literature, $\mathrm{n}^{\circ}$ XLIII (1), p. 108-120.

SINAI,A. (2010): "The business cycle in a changing economy: Conceptualization, measurement, dating", American Economic Review: Papers \& Proceedings, $\mathrm{n}^{\circ} .100$, p. $25-29$.

STÖRHR, W. B. (1990): Global Challenge and Local Response, London, Mansell.

TAMAMES, R. (2009): Para salir de la crisis global. Análisis y soluciones, Madrid, Editorial EDAF.

TAYLOR, P. J. (2004): World City Network: A Global Urban Analysis, London, Routledge.

VÁZQUEZ BARQUERO, A. (2011): "Los territorios innovadores, espacios estratégicos del desarrollo", en Curbelo, J. L., Parrilli, M.D. y Alburquerque, F. (coord.) Territorios innovadores y competitivos, San Sebastián, Publicaciones Orkestra, p. 75-88.

VAZQUEZ BARQUERO, A. (2010): The new forces of development. Territorial policy for endogenous development, Singapore, World Scientific.

VÁZQUEZ BARQUERO, A. (1999): Desarrollo, redes e innovación, Madrid, Pirámide.

VAZQUEZ BARQUERO, A. (1993): Política económica local, Pirámide, Madrid. VÁZQUEZ BARQUERO, A., SEISDEDOS, G. y LACALLE, M.C. (2009): Las regiones policéntricas, territorio estratégico del desarrollo económico, Santiago de Compostela, Xunta de Galicia. 